\title{
Formación bibliotecaria, IFLA y acceso público a información de calidad en Guatemala
}

\section{Library training, IFLA and public access to quality information in Guatemala}

\author{
Jesús Guzmán Domínguez \\ Universidad de San Carlos de Guatemala \\ jguzman@fahusac.edu.gt \\ https://orcid.org/0000-0003-1839-6755 \\ Alice Patricia Burgos Paniagua \\ Universidad de San Carlos de Guatemala \\ aburgos@fahusac.edu.gt
}

\section{Referencia del ensayo}

Guzmán Domínguez, J. y Burgos Paniagua, A. P. (2021). Formación bibliotecaria, IFLA y acceso público a información de calidad en Guatemala. Revista Guatemalteca de Cultura. 1(2). 15-27 DOI 10.46954/revistaguatecultura.v1i2.11

Recibido 15/01/2021

Aceptado 22/06/2021

\section{Resumen}

La relevancia social de la formación profesional de bibliotecarios en la actualidad radica en la función que desempeñan para garantizar el acceso público a la información a través de servicios de información de calidad. Esta función está incluida en las directrices emanadas de la Federación Internacional de Asociaciones de Bibliotecarios y Bibliotecas -IFLA, orientadas a responder a las exigencias profesionales de la sociedad de la información. Por ello, este ensayo ofrece una revisión teórica del 
alcance de los documentos de la IFLA y la relación directa que tienen con la formación de los bibliotecarios y el ejercicio de esta profesión; la demanda de nuevos servicios de información; las competencias requeridas a los técnicos y profesionales de la Bibliotecología para responder a nuevas necesidades de información y la urgencia de trabajar en nuevos perfiles de egreso para las carreras Bibliotecario General y Licenciatura en Bibliotecología.

Palabras clave: profesión bibliotecaria, directrices de la IFLA, desarrollo sostenible, diseño curricular de Bibliotecario General, diseño curricular de Licenciatura en Bibliotecología

\section{Abstract}

The social relevance of the professional training of librarians today lies in the role they play to guarantee public access to information through quality information services. This function is included in the guidelines issued by the International Federation of Library Associations and Libraries -IFLA, aimed at responding to the professional demands of the information society. For this reason, this essay offers a theoretical review of the scope of IFLA documents and the direct relationship they have with the training of librarians and the exercise of this profession; the demand for new information services; the competencies required of Library technicians and professionals to respond to new information needs and the urgency of working on new graduate profiles for the General Librarian and Librarian Bachelor's degrees.

\Keywords: library profession, IFLA guidelines, sustainable development, General Librarian curriculum design, Bachelor of Librarian curriculum design

\section{Intrducción}

La Escuela de Bibliotecología de la Facultad de Humanidades de la Universidad de San Carlos de Guatemala se fundó el 1 de febrero de 1948, dándose inicio con ello a la enseñanza universitaria de la Bibliotecología en Guatemala. Si el objeto de estudio de esta ciencia es la biblioteca entendida como sistema de información, de ello se derivan los cambios significativos que ha tenido posterior a la Segunda Guerra Mundial (1939-1945); el perfeccionamiento de la invención de la computadora, los avances en la programación, 
el surgimiento de la Ciencia de la Información, las redes, los móviles y las tecnologías de la información y la comunicación, transformaron significativamente las bases teóricas y proyecciones de la Bibliotecología.

El conjunto de hechos históricos que se mencionan, originaron una nueva forma de gestionar y difundir la información; hoy día existen múltiples soportes, canales y necesidades de información que usuarios con un nuevo perfil demandan; esos usuarios requieren nuevos servicios de información y como consecuencia, debe actualizarse la formación de los técnicos y profesionales de la Bibliotecología.

En el contexto de la formación universitaria de los técnicos y profesionales de la Bibliotecología y el ejercicio de su labor, actualmente existen muchos aspectos y circunstancias que justifican socialmente el acceso público a la información a través de servicios de calidad, considerados estos como recursos indispensables para la consecución del desarrollo integral de todo ser humano. Esta necesidad que ha cobrado mucha relevancia en la sociedad actual, marcada por grandes desigualdades, pone de manifiesto la conveniencia de formar bibliotecarios con el perfil de egreso requerido para el ejercicio de esta profesión, tomando como base las directrices, lineamientos, manifiestos y estándares propuesto por la Federación Internacional de Asociaciones de Bibliotecarios y Bibliotecas IFLA y el contexto en el cual se desenvuelven los bibliotecarios del país.

\section{Contenido}

\section{Federación Internacional de Asociaciones de Bibliotecarios y Bibliotecas}

La Federación Internacional de Asociaciones de Bibliotecarios y Bibliotecas IFLA es una organización no gubernamental sin fines de lucro, principal portavoz internacional de los profesionales de la Bibliotecología y la Documentación y de los usuarios de los servicios de información documental. Según Pérez -Salmerón (2013), la IFLA

\begin{abstract}
"trabaja para mejorar el acceso a la información y a los recursos que son patrimonio cultural de la comunidad mundial dentro de un vertiginoso cambio en los medios impresos y digitales. Se define a sí misma como la voz global de los profesionales de las bibliotecas y la información, y lo materializa a través de su plan estratégico e iniciativas clave que incluyen el acceso al contenido digital, el liderazgo internacional, la divulgación, el patrimonio cultural y el multilingüismo. Establece una agenda profesional a través del desarrollo de directrices, lineamientos, manifiestos, estándares, publicaciones y eventos alrededor del mundo. El status de IFLA como una organización mundial interesada en los servicios bibliotecarios y de información asegura que la voz de los profesionales de la gestión de la información sea representada formalmente ante organismos internacionales como Naciones Unidas" (párr. 2).
\end{abstract}

\section{Origen y objetivos de la IFLA}

La Federación Internacional de Asociaciones de Bibliotecarios y Bibliotecas (IFLA) se fundó en Edimburgo, Escocia en 1927 (Lacasa Otín y Martínez Esquerra, 2020. P.72). Sus valores principales lo constituyen el respaldo total a los 
principios de libertad de acceso a la información y de expresión y la defensa de las creencias de las personas, comunidades y organizaciones que ejercen el derecho de acceso equitativo a la información documental a través de servicios de calidad. Actualmente la IFLA continúa desempeñando su misión como organización líder en el mundo de la Bibliotecología internacional, que protege los intereses de los bibliotecarios de todo el mundo y promueve la observancia y práctica de los conocimientos sobre la disciplina.

\section{Figura 1}

Estructura de la Federación Internacional de Asociaciones de Bibliotecarios y Bibliotecas.
Asamblea General

Fuente. elaboración propia.

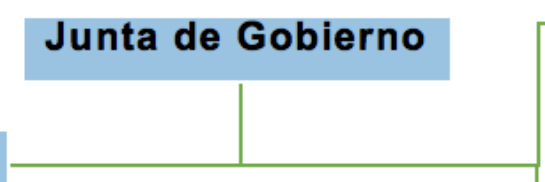

La IFLA es la portavoz de las asociaciones de bibliotecarios, bibliotecas y profesionales de la información a nivel mundial. Sus objetivos son los siguientes:

- Emprender, promover y coordinar investigaciones y estudios.

- Recoger, coleccionar, editar y difundir las informaciones concernientes a las bibliotecas, la bibliografía, la información y formación profesional.

- Organizar reuniones y congresos de carácter general o especializado.

- Colaborar con las organizaciones internacionales en el mundo de la documentación y archivos.
5 divisiones

\section{Programas Estratégicos}

\section{Grupos de Interés Especial}

- Crear una oficina para llevar a cabo tareas específicas y emprender todas las actividades susceptibles de ayuda a la realización de objetivos teóricos y prácticos de la biblioteconomía en su conjunto. (Lacasa Otín y Martínez Esquerra, 2020. P.71).

Para hacer realidad sus objetivos, la IFLA apoya a nivel internacional el proceso de toma de decisiones para la creación, mejoramiento y funcionamiento de bibliotecas y centros de documentación, lo hace a través de la generación de documentos como manifiestos, directrices, pautas, lineamientos, estándares y otras investigaciones de actualidad sobre aspectos técnicos y científicos de la Bibliotecología y los diferentes contextos en donde los profesionales de esta especialidad realizan su labor. 


\section{La IFLA, el desarrollo sostenible y el rol de las bibliotecas}

Resulta sumamente interesante que la Federación Internacional de Asociaciones de Bibliotecarios y Bibliotecas - IFLA-, haya abordado esta temática con énfasis en el desarrollo sostenible desde el año 2002, mediante la Declaración acerca de las bibliotecas y el desarrollo sostenible, realizada en Glasgow, Escocia, el 24 de agosto del año 2002.

- Más importante aún, son las expresiones iniciales de esta Declaración, en donde la IFLA declara, reconoce y afirma:

- Declara que todos los seres humanos tienen el derecho fundamental a un ambiente adecuado para su salud y bienestar.

- Reconoce la importancia de un compromiso con el desarrollo sostenible para satisfacerlas necesidades del presente, sin comprometer las capacidades del futuro.

- Afirma que los servicios de bibliotecas e información promueven el desarrollo sostenible, al asegurar la libertad de acceso a la información. (Federación Internacional de Asociaciones de Bibliotecario y Bibliotecas IFLA, 2002, párr. 2)

De la misma manera, se observa una visión clara del rol que las unidades de información deben desempeñar, para coadyuvar a la implementación de estas propuestas de desarrollo, misma que no ha cambiado:
"Los servicios de bibliotecas e información prestan un apoyo esencial al aprendizaje para toda la vida, a la toma independiente de decisiones y al desarrollo cultural de todos. Mediante sus vastas colecciones y variedad de soportes informacionales ofrecen orientación y oportunidades de aprendizaje. Los servicios de bibliotecas e información ayudan a las personas a mejorar sus competencias educativas y sociales, condiciones imprescindibles en la sociedad de la información y para una participación sostenida en la democracia. Las bibliotecas fomentan los hábitos de lectura, la alfabetización informacional y promueven la educación, el conocimiento público y las oportunidades de capacitación" (Federación Internacional de Asociaciones de Bibliotecario y Bibliotecas IFLA, 2002, Párr. 5).

A pesar de lo expuesto, el compromiso de las unidades de Información propuesto por la IFLA desde el año 2002, no tuvo el efecto esperado en las mismas, ya que en la mayoría de países subdesarrollados el presupuesto para el fortalecimiento de bibliotecas, centros de documentación y archivos es reducido y en un buen número de casos inexistente; es menester reconocer que, la deficiente formación de los profesionales de la información documental y su escaso número, también es un factor de alta incidencia.

Fue de esta manera y ante la inminente finalización de la Agenda de Desarrollo 20002015, que la IFLA retoma con más fuerza el compromiso de las unidades de información documental y la urgencia de comprometerse con las propuestas de desarrollo sostenible. 
La IFLA reunida en Lyon, Francia, en el año 2014, ante la finalización de los ODM, redacta la Declaración de Lyon sobre el Acceso a la Información y el Desarrollo, en donde se puede apreciar una recontextualización del compromiso con las agendas relacionadas con el desarrollo sostenible.

Nosotros, los abajo firmantes, creemos que el progresivo acceso a la información y al conocimiento en toda la sociedad, ayudada por la disponibilidad de las tecnologías de la información y las comunicaciones (TIC), apoya el desarrollo sostenible y mejora la calidad de vida de las personas. (Federación Internacional de Asociaciones de Bibliotecarios y Bibliotecas IFLA, 2014, Párr. 2)

La base de servicios de información de calidad se encuentra en una formación académica de calidad; la misma debe ser inclusiva, actualizada a las demandas de la sociedad del conocimiento y de carácter integral, además de los aspectos epistemológicos propios de estas ciencias.

\section{Tabla 1}

Documentos de la IFLA relacionados con la temática del desarrollo sostenible.

\begin{tabular}{|c|c|c|c|}
\hline No. & Documento & Año & Ciudad \\
\hline 01. & Declaración acerca de las Bibliotecas y el Desarrollo Sostenible & 2002 & Glasgow, Escocia, \\
\hline 02. & $\begin{array}{l}\text { Grupo de Interés Especial sobre la Sostenibilidad } \\
\text { Medioambiental y las Bibliotecas }\end{array}$ & 2011 & San Juan, Puerto Rico \\
\hline 03. & Grupo de Interés Especial sobre los Nuevos Profesionales & 2011 & San Juan, Puerto Rico \\
\hline 04. & Declaración sobre las Bibliotecas y el Desarrollo & 2013 & Singapur \\
\hline 05. & $\begin{array}{l}\text { Declaración de Lyon sobre el acceso a la información y el } \\
\text { desarrollo }\end{array}$ & 2014 & Lyon, Francia \\
\hline 06. & $\begin{array}{l}\text { Guía: Las Bibliotecas y la Agenda de Desarrollo post-2015 de las } \\
\text { Naciones Unidas }\end{array}$ & 2014 & La Haya, Holanda \\
\hline 07. & Las bibliotecas y la implementación de la Agenda 2030 de la ONU & 2015 & La Haya, Holanda \\
\hline 08. & $\begin{array}{l}\text { Las bibliotecas pueden promover la implementación de la Agenda } \\
2030 \text { de la ONU }\end{array}$ & 2015 & La Haya, Holanda \\
\hline 09. & $\begin{array}{l}\text { Acceso y oportunidades para todos, ¿Cómo contribuyen las } \\
\text { bibliotecas a la Agenda } 2030 \text { de la ONU? }\end{array}$ & 2016 & La Haya, Holanda \\
\hline 10. & Declaración de Santiago & 2018 & Santiago, Chile \\
\hline 11. & ¿Cómo las bibliotecas pueden contribuir a la sostenibilidad? & 2018 & La Haya, Holanda \\
\hline 12. & $\begin{array}{l}\text { Manual para contar historias: Las Bibliotecas y los Objetivos de } \\
\text { Desarrollo Sostenible }\end{array}$ & 2018 & La Haya, Holanda \\
\hline 13. & Resumen del Informe de la Visión Global de la IFLA & 2018 & Barcelona, Esp. \\
\hline 14. & $\begin{array}{l}\text { Visión global de la Federación Internacional de Asociaciones e } \\
\text { Instituciones de Bibliotecas: estrategia para el período 2019-2024 }\end{array}$ & 2019 & Atenas, Grecia \\
\hline 15. & Estrategia de la IFLA 2019-2024 & 2019 & La Haya, Holanda \\
\hline
\end{tabular}

Fuente. Elaboración propia. 


\section{Agenda 2030 para el desarrollo sostenible}

\section{¿Qué es la Agenda 2030?}

La Agenda 2030 es la propuesta de la Organización de las Naciones Unidas para continuar con el programa de erradicación de la pobreza en los países subdesarrollados o en vías de desarrollo, como se les conoce en algunos sectores sociales, religiosos y políticos.

Es la continuidad del Programa Objetivos de Desarrollo del Milenio -ODM- (2000-2015) que la misma Organización de las Naciones Unidas propuso en su momento y que una vez concluido el intervalo de tiempo y su respectivo análisis en cuanto a logros y tareas pendientes, replantea a través de los Objetivos de Desarrollo Sostenible - ODS-

Los Objetivos de Desarrollo del Milenio - ODMfueron ocho y los Objetivos de Desarrollo sostenible son 17, la razón principal de este aumento se fundamenta en la cantidad de compromisos que quedaron pendientes en la propuesta de desarrollo 2000-2015, ya que muchos de ellos, propuestos por los gobiernos de los países más pobres no fueron ejecutados y los niveles de pobreza, debido al olvido o abandono de la deuda social, incremento los índices de pobreza en los países subdesarrollados.

La importancia de la Agenda 2030, a través de la implementación de los Objetivos de Desarrollo Sostenible -ODS-, es vital para las agendas de disminución de los niveles pobreza en los países subdesarrollados y la consecución de sociedades más justas, equitativas y solidarias.
Los problemas que enfrentan los países subdesarrollados se han incrementado por la ausencia de la aplicación del ODS número 10, ya que la desigualdad en y entre los países se ha incrementado y la mayoría de los males que enfrenta el tercer mundo, son causados por la inobservancia de los compromisos adquiridos por los países desarrollados.

\section{La IFLA y la Agenda 2030 de la ONU}

En septiembre de 2015, los Estados miembros de las Naciones Unidas adoptaron el documento Transformar nuestro mundo: la Agenda 2030 para el Desarrollo Sostenible, que incluye 17 Objetivos de Desarrollo Sostenible que abarcan el desarrollo económico, ecológico y social.

Previo a la publicación de la Agenda 2030 de la Organización de la Naciones Unidas, la IFLA participó activamente en su creación, abogando por la inclusión del acceso a la información, la salvaguarda del patrimonio cultural, la alfabetización universal y el acceso a las Tecnologías de la Información y la Comunicación TIC. Las bibliotecas son instituciones clave para alcanzar los ODS. (Federación Internacional de Asociaciones de Bibliotecarios y Bibliotecas IFLA, 2016, Párr. 6)

En Guatemala la implementación de esta agenda de desarrollo propuesta por la ONU converge con Plan Nacional de Desarrollo: K'atun Nuestra Guatemala 2032, propuesto por el gobierno de Otto Pérez Molina, el 12 de agosto de 2014. La relación existente entre el Plan Nacional de Desarrollo: K'atun, Nuestra Guatemala 2032 con la Agenda 2030 y los Objetivos de Desarrollo Sostenible es innegable. 
"Al momento de la aprobación, en septiembre de 2015, por parte de la Asamblea General de las Naciones Unidas: Agenda 2030, Guatemala ya contaba con su Plan Nacional de Desarrollo (2014), que contiene 36 prioridades, 80 metas y 730 lineamientos. De esta manera, fue necesario articular la Agenda 2030 y el Plan Nacional de Desarrollo (PND) para así aunar los esfuerzos que se realizarán para cumplir las metas de ambos instrumentos de desarrollo y adecuar la Agenda 2030 a las prioridades, necesidades y posibilidades del país. Por esta razón, el Consejo Nacional de Desarrollo Urbano y Rural lideró durante 2016 un proceso de priorización de la Agenda 2030 a la luz del PND, la Política General de Gobierno (PGG), las metas pendientes de cumplir de los Objetivos de Desarrollo del Milenio (ODM) y otras agendas internacionales. Esta priorización incluyó, además, una etapa de consulta a la ciudadanía y validación, lo que finalmente dio como resultado una agenda priorizada por Guatemala con un contenido de 17 objetivos, 129 metas y 200 indicadores. Secretaría de Planificación y Programación de la Presidencia" (SEGEPLAN, 2016, p. 21).

En este Plan no fue considerado como prioridad de desarrollo el garantizar el acceso público a la información (ODS 16.10), lo cual se explica por el desconocimiento de los esfuerzos realizados por la Federación Internacional de Asociaciones de Bibliotecarios y Bibliotecas -IFLA- en la ONU, para que este derecho fuera considerado como sustantivo para lograr la erradicación de la pobreza y el desarrollo integral de las personas.

\section{La Escuela de Bibliotecología de la Facultad de Humanidades}

Ante las directrices de la IFLA y la Agenda 2030 para el desarrollo sostenible, es indispensable realizar un análisis de los diseños curriculares de las carreras Bibliotecario General y Licenciatura en Bibliotecología que ofrece la Escuela de Bibliotecología de la Facultad de Humanidades de la Universidad de San Carlos de Guatemala. Esto permitiría que sus procesos de enseñanza aprendizaje posibiliten el egreso de técnicos y profesionales de la Bibliotecología con las competencias específicas que les permitan coadyuvar significativamente, en la consecución del desarrollo sostenible a través de servicios que "garanticen el acceso público a la información y a la protección de las libertades fundamentales, de conformidad con las leyes nacionales y los acuerdos internacionales" (Granda y Machin-Mastromatteo, 2016, párr. 7), meta 10 del ODS 16 que empata con la propuesta de formación y trabajo realizada por la Federación Internacional de Asociaciones de Bibliotecarios y Bibliotecas (IFLA).

\section{Servicios de información de calidad}

Desde la Declaración acerca de las bibliotecas y el desarrollo sostenible, realizada en Glasgow, Escocia, en 2002, la IFLA ha hecho énfasis en el compromiso que tienen los técnicos y profesionales de la información documental para implementar acciones que garanticen a los usuarios el ejercicio del derecho de acceso público a la información, lo cual exige del personal responsable de las unidades de información documental, un cambio sustantivo en los servicios de información que se ponen a disposición de los usuarios. 
Con relación a lo expuesto, es menester afirmar que, con la globalización de la información, la expansión de la Web, el uso generalizado de móviles y oportunidades de conectividad, los escenarios se han modificado sustantivamente; lo cual ha dado origen a una nueva definición de servicios, usuarios, soportes y necesidades de información; y con ello también la necesidad de redefinir el perfil de egreso y profesional para estas Carreras.

La IFLA ha definido claramente cuál es el rol que deben desempeñar los profesionales de la información documental que trabajan en bibliotecas, centros de documentación y archivos; el compromiso consiste en adquirir las competencias necesarias, contar con los recursos documentales y los niveles de conectividad requeridos para ofrecer servicios de información de calidad, que incidan positivamente en el desarrollo de los países.

En la presentación del Reporte de Tendencias de 2014, ¿Surcando las olas o atrapados en la marea? Navegando el entorno en evolución de la información, puede leerse la motivación principal de su labor, sus iniciativas claves y el objetivo principal de sus programas profesionales.

"IFLA es la voz global de los profesionales de las bibliotecas y la información (...) Trabajamos para mejorar el acceso a la información y a los recursos que son patrimonio cultural de la comunidad mundial dentro de un vertiginoso cambio en los medios impresos y digitales. Nuestras iniciativas claves incluyen el acceso al contenido digital, el liderazgo internacional, la divulgación, el patrimonio cultural y el multilingüismo. En nuestros programas profesionales construimos la capacidad de nuestros miembros y establecemos la agenda profesional a través del desarrollo de directrices, lineamientos, manifiestos, estándares, publicaciones y eventos alrededor del mundo" (Federación Internacional de Asociaciones de Bibliotecario y Bibliotecas, 2014, párrafos 3 y 4).

La propuesta de formación para técnicos y profesionales de la Bibliotecología que la IFLA desarrolla a través de sus programas profesionales no es reciente, solamente que con el advenimiento de la sociedad de la información y del conocimiento, esta se ha vuelto más integral, ya que cubre todas las áreas actuales de la formación en la especialidad y las habilidades que demanda el nuevo perfil profesional.

Una mirada a algunas de sus publicaciones pone de manifiesto lo afirmado y evidencia la necesidad de que esos contenidos sean considerados en el proceso de formación de los bibliotecarios y otros profesionales de la información. 
Tabla 2

Algunos documentos de IFLA relacionados con la formación y labor de los bibliotecarios.

\begin{tabular}{lll}
\hline No. & \multicolumn{1}{c}{ Documento } & Año \\
\hline 01. & Manifiesto sobre la biblioteca pública & 1994 \\
\hline 02. & Declaración sobre bibliotecas y libertad intelectual & 1999 \\
\hline 03. & Manifiesto por la biblioteca multicultural & 2006 \\
\hline 04. & $\begin{array}{l}\text { Declaración de acceso a la información e identificación de personas en registros } \\
\text { históricos }\end{array}$ & 2008 \\
\hline 05. & Manifiesto sobre transparencia & 2008 \\
\hline 06. & Manifiesto sobre bibliotecas digitales & 2010 \\
\hline 07. & Recomendaciones sobre alfabetización informacional y mediática & 2011 \\
\hline 08. & Código de ética para bibliotecarios y otros profesionales de la información. & 2012 \\
\hline 09. & Manifiesto sobre Internet & 2014 \\
\hline 10. & ¿Surcando las olas o atrapados en la marea? & 2014 \\
\hline 11. & Declaración de privacidad en el entorno bibliotecario & 2015 \\
\hline 12. & Declaración sobre derecho al olvido & 2016 \\
\hline 13. & Declaración sobre alfabetización digital & 2017 \\
\hline 14. & Declaración sobre noticias falsas & 2018 \\
\hline 15. & Directrices para el proyecto de unificación digital & 2019 \\
\hline
\end{tabular}

Fuente. elaboración propia

\section{Las carreras Bibliotecario General y Licenciatura en Bibliotecología}

En Guatemala, la enseñanza universitaria de la Bibliotecología se inició el 1 de febrero de 1948; para 1962 ya se encontraban activas las Carreras de Bibliotecario General y Licenciatura en Bibliotecología. Los planes de estudio de estas Carreras fueron readecuados por última vez en 2008, sobresaliendo la necesidad de profundizar en el aspecto disciplinar y de dar respuesta a las demandas de la profesión en el contexto de la sociedad de la información; sin embargo, en ese momento histórico algunas áreas del conocimiento fueron incluidas con muy poca presencia en los planes de estudio y hoy día, forman parte del cuerpo disciplinar de las Ciencias de la Información Documental, lo cual exige la elaboración de nuevos diseños curriculares o readecuación de los existentes. Pirela Morillo y Peña Vera (2005) lo expresan de la siguiente manera:

"La formación académica-profesional en ciencias de la información debe transitar hacia la introducción del enfoque de competencias, lo cual se enmarca a su vez en el desplazamiento que se está dando en estos momentos de la sociedad de la información a la sociedad del conocimiento y la comunicación, entendida esta última como una sociedad 
que utiliza el aprendizaje permanente como una estrategia de desarrollo personal y social. Esto plantea la vuelta al sujeto, el desarrollo de su cognición y la expansión de su inteligencia, soportado todo ello en el acceso y uso social de la información mediatizada por las tecnologías telemáticas. Ante esta nueva sociedad se requiere que el profesional de la información asuma un rol mediador, que incluye la contribución al aprendizaje de las personas y grupos; la creación de conocimiento útil basado en información, y la creación de contenidos digitales sobre la base de criterios de utilidad, accesibilidad y calidad, pero sin dejar de lado el componente ético que debe orientar la producción y el uso del conocimiento en la cibersociedad" (p. 136).

La importancia estratégica que posee la gestión eficaz y el acceso público a la información para el desarrollo integral de las personas permite vislumbrar la trascendencia de estas Carreras y justifica todas las acciones que se realicen para buscar su desarrollo, actualización y efectividad.

\section{Conclusión}

La IFLA siempre se ha interesado por el desarrollo sostenible, como se evidencia con la Declaración acerca de las Bibliotecas y el Desarrollo Sostenible, de 2002; pero con las propuestas de los Objetivos de Desarrollo del Milenio -ODM- y los Objetivos de Desarrollo Sostenible -ODS- se vino a consolidar el importante rol que las bibliotecas tienen para contribuir en su consecución.
Los documentos de la IFLA evidencian lo que está pendientes en los servicios de información que ofrecen las bibliotecas, la evolución que estos han tenidos en la sociedad del conocimiento y cómo satisfacer las necesidades de información de nuevos usuarios. Los documentos de la IFLA deben incluirse en la formación académica de los bibliotecarios, porque ponen parámetros y paradigmas a alcanzar.

Existen documentos generados por la IFLA para todos los cursos relacionados con la gestión de las bibliotecas: documentos sobre servicios a los usuarios; alfabetización informacional, bibliotecas digitales, educación en los diferentes niveles, servicios para usuarios especiales, uso de Internet, sobre la transparencia, derecho al olvido, noticias falsas, ética de la investigación, derechos de autor, servicios inclusivos de información, procesos técnicos y muchos más.

La situación actual de las carreras Bibliotecario General y Licenciatura en Bibliotecología requiere cambios sustanciales, se debe trabajar en la creación de nuevos perfiles de egreso y de ellos derivar las competencias específicas para cada Carrera y los otros elementos curriculares como los ejes transversales, las áreas curriculares, el plan de estudios, la malla curricular y los contenidos de las asignaturas.

Las multialfabetizaciones constituyen parte fundamental para garantizar el acceso público a la información; entonces la tecnología, el pensamiento crítico, la capacidad de gestión y la elaboración de proyectos son algunas competencias para considerar en el nuevo perfil de egreso y profesional de los bibliotecarios. 


\section{Referencias}

Granda, R. y Machin-Mastromatteo, J. (2016). De Caracas a Lyon: ¿un camino al desarrollo sostenible? Información y desarrollo, 32(2), 216- 218. http:// dx.doi.org/10.1177/0266666915626830

Federación Internacional de Asociaciones de Bibliotecario y Bibliotecas IFLA. (24 de agosto de 2002). Declaración acerca de las bibliotecas y el desarrollo sostenible. https://www.ifla.org/ES/publications/declaraci-n-acerca-de-lasbibliotecas-y-el-desarrollo-sostenible

Federación Internacional de Asociaciones de Bibliotecarios y Bibliotecas IFLA. (18 de Agosto de 2014). Declaración de Lyon sobre el Acceso a la Información y al Desarrollo. https://blogs.ifla.org/lac/2014/08/declaracion-de-lyonversion-en-espanol/

Federación Internacional de Asociaciones de Bibliotecarios y Bibliotecas IFLA. (2014).Reporte de Tendencia 2014: ¿surcando las olas o atrapados en la marea? https://trends.ifla.org/files/trends/assets/ifla-trend-report_spanish. pdf

Federación Internacional de Asociaciones de Bibliotecarios y Bibliotecas IFLA. (18 de Agosto de 2016). Acceso y Oportunidades para Todos: cómo Contribuyen las Bibliotecas a la Agenda 2030 de las Naciones Unidas. https://www.ifla. org/files/assets/hq/topics/libraries-development/documents/access-andopportunity-for-all-es.pdf

Lacasa Otín, M. y Martínez Esquerra, P. (2021). La IFLA, origen, evolución y situación actual.https://dialnet.unirioja.es/servlet/articulo?codigo=224212

Pérez-Salmerón, G. (2013). ¿Surcando las olas o atrapados en la marea? Navegando el entorno en evolución de la información. http://www.ub.edu/ blokdebid/es/content/\%C2\%BFsurcando-las-olas-o-atrapados-en-la-mareanavegando-el-entorno-en-evolucion-de-la-informaci

Pirela Morillo, J. y Pena Vera, T. (205) Nuevos desafíos para la formación del profesional de la información frente al surgimiento de la cibersociedad: un enfoque de competencias. Investigación bibliotecológica. 2005, vol.19, n.3, pp.118-139. http://www.scielo.org.mx/scielo.php?script=sci_ arttext\&pid=S0187- 358X2005000100007\&lng=es\&nrm=iso.

Secretaría de Planificación y Programación de la Presidencia [SEGEPLAN]. (2021, 15 de mayo). Metodología para la integración de la Agenda 2030 en la planificación nacional mediante la identificación de eslabones y nodos críticos. https://www.segeplan.gob.gt/nportal/index.php/ods 


\section{$\angle$ Sobre los autores}

\section{Jesús Guzmán Domínguez}

Es estudiante de la Maestría en Currículum, Licenciado en Bibliotecología y Bibliotecario General, por la Universidad de San Carlos de Guatemala y Profesor de Enseñanza Media en Filosofía por la Universidad Francisco Marroquín. Ha escrito artículos sobre Bibliotecología y Educación. Actualmente es director de la Escuela de Bibliotecología y docente titular de la Facultad de Humanidades de la Universidad de San Carlos de Guatemala.

\section{Alice Patricia Burgos Paniagua}

Es Doctora en Educación, Coordinadora del Doctorado en Educación y docente en diferentes cursos e Investigadora de la Facultad de Humanidades de la Universidad de San Carlos de Guatemala.

\section{Copyright (c) Jesús Guzmán Domínguez y Alice Patricia Burgos Paniagua}

Este texto está protegido por una licencia Creative Commons 4.0. CC

Usted es libre para compartir, copiar y redistribuir el material en cualquier medio o formato y adaptar el documento, remezclar, transformar y crear a partir del material para cualquier propósito, incluso comercialmente, siempre que cumpla la condición de atribución: usted debe reconocer el crédito de una obra de manera adecuada, proporcionar un enlace a la licencia, e indicar si se han realizado cambios. Puede hacerlo en cualquier forma razonable, pero no de forma tal que sugiera que tiene el apoyo del licenciante o lo recibe por el uso que hace. 